\title{
Genetic Algorithm Based Solution of Fuzzy Multi-Objective Transportation Problem
}

\author{
Jaydeepkumar M. Sosa \\ Department of Mathematics, \\ Sardar Vallabhbhai National Institute of Technology, Surat, Gujarat, India. \\ Corresponding author: jaydeepkumarworld@gmail.com \\ Jayesh M. Dhodiya \\ Department of Mathematics, \\ Sardar Vallabhbhai National Institute of Technology, Surat, Gujarat, India. \\ E-mail: jdhodiya2002@yahoo.com
}

(Received May 5, 2020; Accepted August 11, 2020)

\begin{abstract}
Optimizing problems in the modern era, the single objective optimization problems are insufficient to hold the full data of the problem. Therefore, multi-objective optimization problems come to the rescue. Similarly, in daily life problems, the parameters used in the optimization problem are not always fixed but there may be some uncertainty and it can characterize by fuzzy number. This work underlines the genetic algorithm (GA) based solution of fuzzy transportation problem with more than one objective. With a view to providing the multifaceted choices to decision-maker (DM), the exponential membership function is used with the decision-makers desired number of cases which consisted of shape parameter and aspiration level. Here, we consider the objective functions which are non-commensurable and conflict with each other. To interpret, evaluate and exhibit the usefulness of the proposed method, a numerical example is given.
\end{abstract}

Keywords- Fuzzy optimization, GA, Exponential membership function, Decision-maker (DM).

\section{Introduction}

Normally the classical transportation problem (TP) was mostly utilized while taking the best decision in business and management and according to Verma et al. (1997), its structure is same as a linear programming problem (LPP). The model related to transportation, first developed by Kantorovich (1960) and Hitchcock (1941). According to Chanas et al. (1984), the transportation problem is associated to transport commodity initially located at different sources to different destination. Because of its structure, transportation problem is likely to be altered over to standard LPP and according to Verma et al. (1997) solution can be derived with the help of the simplex method. Intending to find the superior solution for the TP, the modified distribution (MODI) Method is beneficial.

In real life transportation scenario objective function is not only a single, but there may be more than one conflicting objectives. Hence, its study and finding a better and optimal solution is always required. Charnes and Cooper (1954) first gives the solution of a managerial type of TP involving more than one conflicting objective. Lee and Moore (1973) study and obtained the solution of multi-objective transportation problem (MOTP) with a goal programming approach. Garfinkel et al. (1974) provided a solution of MOTP by giving low and high priorities to objectives. Das et al. (1999) studied the fuzzy programming approach and gives the solution of MOTP with interval form parameters. Mahapatra et al. (2010) studied the solution Fuzzy multi-objective transportation problem (FMOTP) with various stochastic environments. Maity and Roy (2014) presented the 
International Journal of Mathematical, Engineering and Management Sciences

Vol. 5, No. 6, 1452-1467, 2020

https://doi.org/10.33889/IJMEMS.2020.5.6.108

solution of MOTP with multi choice cost using the utility function approach. They first convert the multi-choice parameter into the real parameter and then solve this problem. In a Transportation problem, the required parameters such as availability, demand, cost, etc., do not always be fixed number but varies according to the circumstances hence a fuzzy number or an interval number is required to represent this uncertainty.

Zimmermann (1978) studied MOTP and provided its solution by fuzzy programming technique. Many researches (Bit et al., 1993; Jimenez and Verdegay, 1998; Rani and Gulati, 2016) etc. in the study of multi-objective solid transportation problem (MOSTP) and its solution with fuzzy programming approach. With Fuzzy technique, Li and Lai (2000) and Abd El-Wahed (2001) resolve the MOTP. Abd El-Wahed and Lee (2006) and Osuji et al. (2014) gives the solution of FMOTP with the help of goal programming method.

Best of my knowledge, almost proposed method uses a balance type fuzzy programming model and fail for the unbalance model. The solution of such an unbalanced model can be acquired with the GA based hybrid approach. GA works on the base of natural selection moreover, constrained and unconstrained both types of the optimization problem can be solved. Gen et al. (1999) give an efficient solution of the MOTP using GA with spanning tree-based encoding.

GA is developed based on mutation, selection and some other operator and easy to implement as compared to the traditional method. Rather than working with variables like the traditional method, GA will work with variables string-coding represent the solution say Tabassum and Mathew (2014). As GA only need function values at different discrete points, the discontinuous function can also be regulated without any additional burden. GA emphasizes the good information previously found through the use of reproductive operator and adaptively propagated by mutation operator and crossover operator while the traditional method does not effectively use the information obtained.

Here, we presented the GA based hybrid approach and the best effective solution of FMOTP using it. Also, to give more feasibility to DM for batter decision, the exponential membership function which uses the combination of shape parameter and aspiration level provided multiple alternative solutions to the DM to gain better decision for their benefits. We provided the number of solutions under different estimation using GA based approach with different shape parameter and various ranges of aspiration level. This method provides an effective solution of fuzzy type TP, handles the situations of the problem effectively and give a higher degree of satisfaction to the objective functions.

\section{Mathematical Model}

The idea behind the TP is to find out the transportation schedule for which the objectives related to the problem should be optimized. Consider there are total $u$ number of supply centres with available capacity $a_{1}, a_{2}, \ldots, a_{u}$ respectively for transportation and $v$ destinations with required demand $b_{1}$, $b_{2}, \ldots, b_{v}$ respectively, to receive. Let $c_{p q}^{\widetilde{k}}$ is the fuzzy penalty cost related to transport a single unit of a material from $p^{\text {th }}$ supply centre to $q^{\text {th }}$ destination for $k^{\text {th }}$ objective function and $x_{p q}$ be the unknown amount of material transported from $p^{\text {th }}$ supply centre to $q^{\text {th }}$ destination. The mathematical model with the above notations is underlying as Mode-1. 
International Journal of Mathematical, Engineering and Management Sciences

Vol. 5, No. 6, 1452-1467, 2020

https://doi.org/10.33889/IJMEMS.2020.5.6.108

\section{Model-1}

Minimize $z_{k}=\sum_{p=1}^{u} \sum_{q=1}^{v} \widetilde{c_{p q}^{k}} x_{p q}, k=1,2, \ldots$

Subject to,

$\sum_{q=1}^{v} x_{p q}=a_{p}, p=1,2, \ldots, u$.

$\sum_{p=1}^{u} x_{p q}=b_{q}, q=1,2, \ldots, v$

Also, $x_{p q} \geq 0$, for all $p$ and $q$.

The Model-1 contains $p+q$ constraints and $p q$ decision variables. For the solution of Model-1, its coefficient must be in the realistic form. For this, we need to discuss some preliminaries.

\section{Preliminaries}

\subsection{Positive Ideal Solution (PIS) and Negative Ideal Solution (NIS)}

PIS is defined as the objective function's minimum value, and the objective function's maximum value is the NIS. It took for each objective function to determine the value of exponential membership function.

\subsection{Membership Function}

The data related to the given problem can be normalized with the help of fuzzy exponential membership function $\mu_{z_{k}}(x)$. If the number $z_{k}^{P I S}$ and $z_{k}^{N I S}$ stand for the PIS and NIS respectively for objective $z_{k}$, then $\mu_{z_{k}}(x)$ is represented by as under.

$\mu_{z_{k}}(x)=\left\{\begin{array}{lc}1, & \text { if } z_{k} \leq z_{k}^{\mathrm{PIS}} \\ \frac{e^{-\mathrm{S} \psi_{k}(x)}-e^{-S}}{1-e^{-S}} ; & \text { if } z_{k}^{\mathrm{PIS}}<z_{k}<z_{k}^{\mathrm{NIS}} \\ 0, & \text { if } \mathrm{z}_{k} \geq z_{k}^{\mathrm{NIS}}\end{array}\right\}$

where, $\psi_{k}(x)=\frac{z_{k}-z_{k}^{\mathrm{PIS}}}{z_{k}^{\mathrm{NIS}}-z_{k}^{\mathrm{PIS}}}, 0 \leq \mu_{z_{k}}(x) \leq 1$ and $s \neq 0$, the DM's shape parameter. Moreover, the membership function will be concave and convex accordingly for $s>0$ and $s<0$ in $\left[z_{k}^{P I S}, z_{k}^{N I S}\right]$.

\subsection{Possibilistic Programming Approach}

According to Mahapatra et al. (2010), Dhodiya and Tailor (2016), insufficient information on realworld condition is a crucial concern, since it generates a high degree of uncertainty. Although previous data are given, in the future, the overall performance of the parameters no longer necessarily satisfies the previous model. So, use the fuzzy number to represent this uncertain model to handle this situation with the problem concerned. The definition of probability lies in such a way that, through human choice, a significant part of the knowledge offers and relies on possibilistic in nature. The possibility distribution is expected in the derived form of insufficient data and knowledge of DM and, therefore, the FMOTP model is transformed into a crisp MOTP model through a possibilistic approach say Mahapatra et al. (2010). 
International Journal of Mathematical, Engineering and Management Sciences

Vol. 5, No. 6, 1452-1467, 2020

https://doi.org/10.33889/IJMEMS.2020.5.6.108

\subsection{Triangular Possibilistic Distribution (TPD)}

Its easiness and statistical effectiveness in acquiring the facts, the TPD is generally used for the ambiguous existence of indefinite parameters say Mahapatra et al. (2010). The triangular fuzzy number is a case to represent the fuzzy number. We use its minimum, maximum and mean values to describe the nature of the triangular distribution. The decisive criteria and the qualitative criteria will address several uncertain problems, as well as practical applications. According to Gupta and Mehlawat (2013), the objective function's value is represented in three-place, towards which the cost of moving the three TPD positions to the left side is minimized because the co-ordinates of the vertical point are constant by 0 or 1 . Thus the closing three horizontal coordinates are considered as shown in Figure 1 below.

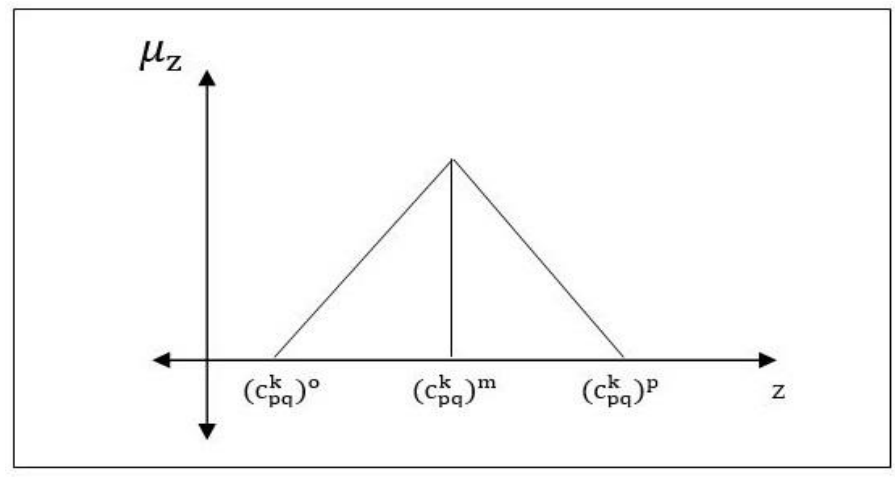

Figure 1. Triangular possibilistic distribution

\section{5 $\alpha$-Level Set}

In fuzzy set theory, the $\alpha$-cut is the most crucial ideas amongst the standout, created by Zadeh (1975). This approach makes use of the fuzzy set idea to create uncertainty within parameters. The $\alpha$-level illustrates the DM's sure bet with respect its fuzzy result, which can also be known as the point of certainty. In a fuzzy set theory largest value yield, a smaller but more confident result in which lower bound and upper bound have a higher membership value.

\section{Formulation of Auxiliary Multi-Objective Transportation Programming Model}

Using TPD idea the $k^{t h}$ vague objective $\widetilde{z_{k}}$ with fuzzy cost $\widetilde{c_{u v}^{k}}$ corresponding to Model-1 can be written as,

$$
\begin{aligned}
& \min \widetilde{\mathrm{z}_{\mathrm{k}}}=\min \left(\sum_{\mathrm{q}=1}^{\mathrm{v}} \sum_{\mathrm{p}=1}^{\mathrm{u}} \widetilde{\mathrm{c}_{\mathrm{pq}}^{\mathrm{k}}} \mathrm{x}_{\mathrm{pq}}\right) \\
& =\min \left(\sum_{\mathrm{q}=1}^{\mathrm{v}} \sum_{\mathrm{p}=1}^{\mathrm{u}}\left(\mathrm{C}_{\mathrm{pq}}^{\mathrm{k}}\right)^{0} \mathrm{x}_{\mathrm{pq}}, \sum_{\mathrm{q}=1}^{\mathrm{v}} \sum_{\mathrm{p}=1}^{\mathrm{u}}\left(\mathrm{C}_{\mathrm{pq}}^{\mathrm{k}}\right)^{\mathrm{m}} \mathrm{x}_{\mathrm{pq}}, \sum_{\mathrm{q}=1}^{\mathrm{v}} \sum_{\mathrm{p}=1}^{\mathrm{u}}\left(\mathrm{C}_{\mathrm{pq}}^{\mathrm{k}}\right)^{\mathrm{p}} \mathrm{x}_{\mathrm{pq}}\right) \\
& =\min \left(\mathrm{z}_{\mathrm{k}}^{0}, \mathrm{z}_{\mathrm{k}}^{\mathrm{m}}, \mathrm{z}_{\mathrm{k}}^{\mathrm{p}}\right) \\
& =\min \left(\mathrm{z}_{\mathrm{k} 1}, \mathrm{z}_{\mathrm{k} 2}, \mathrm{z}_{\mathrm{k} 3}\right)
\end{aligned}
$$

where $\widetilde{\mathrm{c}_{\mathrm{pq}}}=\left(\left(\mathrm{C}_{\mathrm{pq}}^{\mathrm{k}}\right)^{0},\left(\mathrm{C}_{\mathrm{pq}}^{\mathrm{k}}\right)^{\mathrm{m}},\left(\mathrm{C}_{\mathrm{pq}}^{\mathrm{k}}\right)^{\mathrm{p}}\right)$. The numerical representation for $\widetilde{\mathrm{p}_{\mathrm{pq}}}$ defines as under. 
$\left(c_{\mathrm{pq}}^{\mathrm{k}}\right)_{\alpha}^{0}=\mathrm{c}_{\mathrm{pq}}^{0}+\alpha\left(\left(\mathrm{c}_{\mathrm{pq}}^{\mathrm{k}}\right)^{\mathrm{m}}-\mathrm{c}_{\mathrm{pq}}^{0}\right)$

$\left(c_{p q}^{k}\right)_{\alpha}^{m}=\left(c_{p q}^{k}\right)^{m}$

$$
\left(c_{\mathrm{pq}}^{\mathrm{k}}\right)_{\alpha}^{\mathrm{p}}=\left(\mathrm{c}_{\mathrm{pq}}^{\mathrm{k}}\right)^{\mathrm{p}}+\alpha\left(\left(\mathrm{c}_{\mathrm{pq}}^{\mathrm{k}}\right)^{\mathrm{p}}-\left(\mathrm{c}_{\mathrm{pq}}^{\mathrm{k}}\right)^{\mathrm{m}}\right)
$$

The above equation (10) to (12) related to the optimistic, the most likely and pessimistic situation respectively. Now, the FMOTP is translated into a fresh crisp multi-objective transportation problem (CMOTP) as Model-2 below.

\section{Model-2}

$$
\begin{aligned}
& \left(\min \mathrm{z}_{11}, \min \mathrm{z}_{12}, \min \mathrm{z}_{13}, \min \mathrm{z}_{21}, \min \mathrm{z}_{22}, \min \mathrm{z}_{23}, \ldots, \min \mathrm{z}_{\mathrm{K} 1}, \quad \min \mathrm{z}_{\mathrm{K} 2}, \min \mathrm{z}_{\mathrm{K} 3}\right) \\
& =\left(\sum_{\mathrm{q}=1}^{\mathrm{v}} \sum_{\mathrm{p}=1}^{\mathrm{u}}\left(\mathrm{c}_{\mathrm{pq}}^{1}\right)_{\alpha}^{\mathrm{o}} \mathrm{x}_{\mathrm{pq}}, \sum_{\mathrm{q}=1}^{\mathrm{v}} \sum_{\mathrm{p}=1}^{\mathrm{u}}\left(\mathrm{c}_{\mathrm{pq}}^{1}\right)_{\alpha}^{\mathrm{m}} \mathrm{x}_{\mathrm{pq}}, \sum_{\mathrm{q}=1}^{\mathrm{q}} \sum_{\mathrm{p}=1}^{\mathrm{p}}\left(\mathrm{c}_{\mathrm{pq}}^{1}\right)_{\alpha}^{\mathrm{p}} \mathrm{x}_{\mathrm{pq}},\right. \\
& \sum_{\mathrm{q}=1}^{\mathrm{v}} \sum_{\mathrm{p}=1}^{\mathrm{u}}\left(\mathrm{c}_{\mathrm{pq}}^{2}\right)_{\alpha}^{\mathrm{o}} \mathrm{x}_{\mathrm{pq}}, \sum_{\mathrm{q}=1}^{\mathrm{v}} \sum_{\mathrm{p}=1}^{\mathrm{u}}\left(\mathrm{c}_{\mathrm{pq}}^{2}\right)_{\alpha}^{\mathrm{m}} \mathrm{x}_{\mathrm{pq}}, \sum_{\mathrm{q}=1}^{\mathrm{v}} \sum_{\mathrm{p}=1}^{\mathrm{u}}\left(\mathrm{c}_{\mathrm{pq}}^{2}\right)_{\alpha}^{\mathrm{p}} \mathrm{x}_{\mathrm{pq}} \\
& \left.\sum_{\mathrm{q}=1}^{\mathrm{v}} \sum_{\mathrm{p}=1}^{\mathrm{u}}\left(\mathrm{c}_{\mathrm{pq}}^{\mathrm{K}}\right)_{\alpha}^{\mathrm{o}} \mathrm{x}_{\mathrm{pq}}, \sum_{\mathrm{q}=1}^{\mathrm{v}} \sum_{\mathrm{p}=1}^{\mathrm{u}}\left(\mathrm{c}_{\mathrm{pq}}^{\mathrm{K}}\right)_{\alpha}^{\mathrm{m}} \mathrm{x}_{\mathrm{pq}}, \sum_{\mathrm{q}=1}^{\mathrm{v}} \sum_{\mathrm{p}=1}^{\mathrm{u}}\left(\mathrm{c}_{\mathrm{pq}}^{\mathrm{K}}\right)_{\alpha}^{\mathrm{p}} \mathrm{x}_{\mathrm{pq}}\right)
\end{aligned}
$$

Subject to,

$$
\begin{aligned}
& \sum_{\mathrm{q}=1}^{\mathrm{v}} \mathrm{x}_{\mathrm{pq}}=\mathrm{a}_{\mathrm{p}}, \mathrm{p}=1,2, \ldots, \mathrm{u} . \\
& \sum_{\mathrm{p}=1}^{\mathrm{u}} \mathrm{x}_{\mathrm{pq}}=\mathrm{b}_{\mathrm{q}}, \mathrm{q}=1,2, \ldots, \mathrm{v} . \\
& \text { Also, } \mathrm{x}_{\mathrm{pq}} \geq 0, \text { for all } \mathrm{p} \text { and } \mathrm{q} .
\end{aligned}
$$

Model-2 contains all the given objective functions and constraints in a realistic form. The solution approach with GA discussed as follows.

\section{Solution Method for an Auxiliary Model}

It is very imperative to gain the solution of any optimization problem which can reflect reliable judgment of the DMs. To get desirable results that can fulfil the DMs demand, the aspiration level should be selected accordingly. The attitude and requirements of DM are changed during the decision-making process hence the aspiration level is not decided with any consistency. One can determine the output using hyperbolic, exponential, linear and so on membership functions that describe the different aspiration levels of DM say Mahapatra et al. (2010). As DM can choose the degree of satisfaction, the exponential membership function representation the reality better than a linear membership function say Gupta and Mehlawat (2013).

We say GA is converged if after getting some particular solution i.e. optimum value, the values of the objectives remain the same if we passed from one iteration to others. GA has converged at a global optimum for an NP-hard problem is impossible, unless you have a test data set for which the best solution is already known. Moreover, the dimension of the problem so affects the convergence of GA. One can define the size of the chromosome (number of solution) based on the problem's 
International Journal of Mathematical, Engineering and Management Sciences

Vol. 5, No. 6, 1452-1467, 2020

https://doi.org/10.33889/IJMEMS.2020.5.6.108

parameter. Also, one should note that increasing the size of the chromosome affect the GA's rate of convergence.

The Solution of FMOTP using the GA based approach.

The step involves in GAs for the solution of FMOTP are described as under.

(1) Using the TPD formulate the auxiliary model for different $\alpha$-cut.

(2) Find out the PIS and NIS value for $\mathrm{z}_{\mathrm{ki}}(\mathrm{x})$ for $\mathrm{k}=1,2, \ldots, \mathrm{K}$ and $\mathrm{i}=1,2,3$.

(3) Calculate the objective value $\mathrm{z}_{\mathrm{ki}}(\mathrm{x})$ for $\mathrm{k}=1,2, \ldots, \mathrm{K}$ and $\mathrm{i}=1,2,3$.

(4) Using (5), calculate $\mu_{z_{\mathrm{ki}}}(\mathrm{x})$ for $\mathrm{k}=1,2, \ldots, \mathrm{K}$ and $\mathrm{i}=1,2,3$.

(5) The fuzzy membership function is including with the help of product operator, hence the single optimization problem of the above FMOTP can be written as Model-3.

\section{Model-3}

Maximize $\mathrm{w}=\prod_{\mathrm{k}=1}^{\mathrm{K}} \prod_{\mathrm{i}=1}^{3} \mu_{\mathrm{z}_{\mathrm{ki}}}(\mathrm{x})$

Subject to, Eq. (2) to (3) and

$\mu_{\mathrm{k}}(\mathrm{x})-\overline{\mu_{\mathrm{k}}}(\mathrm{x}) \geq 0 ; \mathrm{k}=1,2, \ldots, \mathrm{K}$.

where $\overline{\mu_{\mathrm{ki}}}(\mathrm{x})$ is the DM's aspiration level for which the above Model-3 will be solved.

(6) For the solution of Model-3, The GA applies with the following steps I to VII.

\section{Chromosome Encoding}

Here we use the spanning tree-based prufer number encoding given by Gen et al. (1999). The TP involving $p$ origins and $q$ destinations, its solution contains $p+q-1$ basic cell in transportation tableau. These cells are linearly independent and so they cannot contain any cycle, so it can be considered as a tree (transportation tree). If we denote the origin and destination as nodes or vertices of the tree, then there are a separable set of these vertices. There is $p+q$ vertices connected with edges in the transportation tree. If we consider the origins as nodes of one set and destinations as nodes of another set and drawing the edge between these nodes of these two set if there is an allocation between them, then we find a spanning tree with $p+q$ vertices and $p+q-1$ edge. We use the following step to find prefer number code given by Gen et al. (1999), which works as a chromosome in GA.

Procedure to write prefer number from given spanning tree.

We use the following step to write prefer number from any spanning tree:

(a) Let $i$ be the pendant vertex with lowest-numbered connected by an edge with $j$ numbered pendant vertex in spanning tree. Then the code for GA contains the first number as $j$.

(b) Eliminate the vertex $i$ and edge $(i, j)$ from the given spanning-tree.

(c) Find the next lowest numbered pendant vertex and similarly built the code by writing the vertex number right to $j$. 
International Journal of Mathematical, Engineering and Management Sciences

Vol. 5, No. 6, 1452-1467, 2020

https://doi.org/10.33889/IJMEMS.2020.5.6.108

(d) If only two vertexes remaining then stop otherwise repeat step (a) to (c). To find the spanningtree related to given prefer number, we use the following steps.

Procedure to drawn spanning tree from given prefer number.

(a) For the given prufer number $P$, let $\bar{P}$ be the set contains nodes not appear in $P$ and considered as appropriate for concern.

(b) Repeat the following step (i-ii) until no digit left in $P$.

(i) Let $j$ become the left-most digit of $P$ and $i$ be the lowest-numbered eligible vertex in $\bar{P}$.

(ii) Connect the vertexes $i$ and $j$ by an edge in tree $T$, if both are not contained in $P$ or $\bar{P}$ otherwise instead of $j$ connect the vertexes $i$ and $k$ in tree $T$, after choosing $k$ from $P$ not included in the same set with $i$.

\section{Encoding}

The chromosome is the representation of a solution in the form of encoding. For generating the solution of the FMOTP chromosome must be considered. First, we generate genes on a chromosome with all 0 's, and after we randomly generate the chromosome encoding of a given size.

\section{Fitness Function}

A fitness function calculates the numerical value of the given individual of a chromosome that is it fits the data to the numerical value. With satisfying all the constraints of the model, the fitness function is evaluated.

\section{Selection}

For considering the best solution from the given set of the solution selection process used. It will produce a new solution with more fitness from the given randomly selected solution. It should be noted here the selected solution with higher fitness will produce a new solution with greater probability to fit.

In this problem, we apply tournament selection because tournament selection is very easy to apply with sufficient efficiency. In this step, GA selects randomly $n$ number of solutions from the set of solution and compare each other for the new solution. Comparison gives the difference between this chromosome and so with higher fitness value selected first and so on. We repeated this process until we get the total number of chromosome equal to the size of the population.

\section{Crossover}

Next important step after selection in GA was a crossover. Same like selection operator, In Crossover operator two parents share genetic information to produce new offspring. Many crossover operators are used according to the encoding of a chromosome. In this paper, we used a one-point crossover. It is one of the simple crossover techniques used for random GA process. In a one-point crossover, a common point was selected between two-parent and genes are transformed between them. At the end of the one-point crossover, the more effective child can be obtained, if the good genetic material between the participated parents was transferred to each other.

\section{Mutation}

The mutation is the operator by which the chromosome was modified and sent for the further 
generation to come. Missing genetic information was recovered by a mutation operator. Typically, offspring are muted after recombination has been created. We use inversion mutation for FMOTP solution. Because by this mutation one can preserve the nature of over chromosome. The chromosome string reversed in inverse mutation.

\section{Termination Criteria}

Initially, we have to enter the number of iterations, the algorithm will automatically stop and display the required optimal solution after reaching it.

\section{Numerical Example}

In a company, there are three construction machines $\mathrm{M}_{1}, \mathrm{M}_{2}$ and $\mathrm{M}_{3}$, which can produce 8,19 and 17 units of a commodity, respectively. These quantities were shipped to four required locations $R_{1}$, $R_{2}, R_{3}$ and $R_{4}$ with requirements $11,3,14$ and 16 units respectively. The fuzzy transportation cost $\mathrm{z}_{1}$ and required time $\mathrm{z}_{2}$ for that are as follows.

$$
\begin{aligned}
& z_{1}=\left[\begin{array}{cccc}
(0.5,1,1.3) & (1.3,2,2.1) & (5,7,7.6) & (6,7,7.2) \\
(0.7,1,1.6) & (8.3,9,9.6) & (2.4,3,3.1) & (3,4,4.6) \\
(7.5,8,8.2) & (8.1,9,9.4) & (3.6,4,4.9) & (5.1,6,6.5)
\end{array}\right],
\end{aligned}
$$

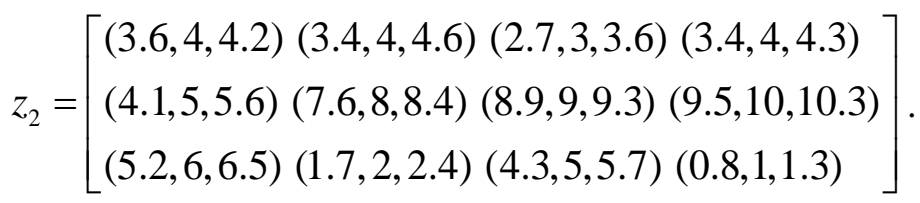

We apply the TDP approach to treat with the fuzzy objectives. Corresponding to $\alpha=0.1$, the mathematical crisp model is as under.

$$
\begin{aligned}
\text { Model-4 } & \begin{aligned}
\min z_{11}= & 0.55 x_{11}+1.37 x_{12}+5.2 x_{13}+6.1 x_{14}+0.73 x_{21}+8.37 x_{22}+2.46 x_{23}+3.1 x_{24}+ \\
& 7.55 x_{31}+8.19 x_{32}+3.64 x_{33}+5.19 x_{34} \\
\min z_{12}= & 1.13 x_{11}+2.01 x_{12}+7.06 x_{13}+7.02 x_{14}+1.06 x_{21}+9.06 x_{22}+3.01 x_{23}+4.06 x_{24}+ \\
& 8.02 x_{31}+9.04 x_{32}+4.09 x_{33}+6.05 x_{34} \\
\min z_{13}= & 1.33 x_{11}+2.11 x_{12}+7.66 x_{13}+7.22 x_{14}+1.66 x_{21}+9.66 x_{22}+3.11 x_{23}+4.66 x_{24}+ \\
& 8.22 x_{31}+9.44 x_{32}+4.99 x_{33}+6.55 x_{34} \\
\min z_{21}= & 3.64 x_{11}+3.6 x_{12}+2.73 x_{13}+3.46 x_{14}+4.19 x_{21}+7.64 x_{22}+8.91 x_{23}+9.55 x_{24}+ \\
& 5.28 x_{31}+1.73 x_{32}+4.37 x_{33}+0.82 x_{34}
\end{aligned}
\end{aligned}
$$




$$
\begin{aligned}
\min z_{22}= & 4.02 x_{11}+4.06 x_{12}+3.06 x_{13}+4.03 x_{14}+5.06 x_{21}+8.04 x_{22}+9.03 x_{23}+10.03 x_{24}+ \\
& 6.05 x_{31}+2.04 x_{32}+5.07 x_{33}+1.03 x_{34} \\
\min z_{23}= & 4.22 x_{11}+4.66 x_{12}+3.66 x_{13}+4.33 x_{14}+5.66 x_{21}+8.44 x_{22}+9.33 x_{23}+10.33 x_{24}+ \\
& 6.55 x_{31}+2.44 x_{32}+5.77 x_{33}+1.33 x_{34}
\end{aligned}
$$

Subject to,

$\sum_{\mathrm{q}=1}^{4} \mathrm{x}_{1 \mathrm{q}} \leq 8, \quad \sum_{\mathrm{q}=1}^{4} \mathrm{x}_{2 \mathrm{q}} \leq 19, \quad \sum_{\mathrm{q}=1}^{4} \mathrm{x}_{3 \mathrm{q}} \leq 17, \quad \sum_{\mathrm{p}=1}^{3} \mathrm{x}_{\mathrm{p} 1} \geq 11, \quad \sum_{\mathrm{p}=1}^{3} \mathrm{x}_{\mathrm{p} 2} \geq 3$,

$\sum_{\mathrm{p}=1}^{3} \mathrm{x}_{\mathrm{p} 3} \geq 14, \sum_{\mathrm{p}=1}^{3} \mathrm{x}_{\mathrm{p} 4} \geq 16$

and $x_{p q} \geq 0$, for all $p=1,2,3$ and $q=1,2,3,4$.

\section{GA Based Model-5}

Maximize $\mathrm{w}=\prod_{\mathrm{k}=1}^{2} \prod_{\mathrm{i}=1}^{3} \mu_{\mathrm{z}_{\mathrm{ki}}}(\mathrm{x})$

Subject to,

Equation (19) to (20) and $\mu_{\mathrm{k}}(\mathrm{x})-\overline{\mu_{\mathrm{k}}}(\mathrm{x}) \geq 0, \mathrm{k}=1,2$.

Similarly, the Model corresponding to $\alpha=0.5$ and $\alpha=0.9$ can be written. To calculate the exponential membership value corresponding to each objective, we required the PIS and NIS value. Table 1 offers the PIS and NIS value for every objective function corresponding to different $\alpha$-cut.

Table 1. PIS and NIS value corresponding to different $\alpha$-cut

\begin{tabular}{|c|l|c|c|c|c|c|c|}
\hline $\boldsymbol{\alpha}-$ Level & \multicolumn{1}{|c|}{ Solution } & $\mathbf{z}_{\mathbf{1 1}}$ & $\mathbf{z}_{\mathbf{1 2}}$ & $\mathbf{z}_{\mathbf{1 3}}$ & $\mathbf{z}_{\mathbf{2 1}}$ & $\mathbf{z}_{\mathbf{2 2}}$ & $\mathbf{z}_{\mathbf{2 3}}$ \\
\hline \multirow{2}{*}{$\alpha=0.1$} & PIS & 118.07 & 143 & 173.03 & 151.52 & 167 & 188.12 \\
\cline { 2 - 8 } & NIS & 228.74 & 265 & 285.24 & 288.31 & 310 & 332.22 \\
\hline \multirow{2}{*}{$\alpha=0.5$} & PIS & 129.15 & 143 & 179.4 & 158.4 & 167 & 195.86 \\
\cline { 2 - 8 } & NIS & 243.7 & 265 & 294.7 & 297.95 & 310 & 340.33 \\
\hline \multirow{2}{*}{$\alpha=0.9$} & PIS & 140.23 & 143 & 185.64 & 307.59 & 167 & 203.48 \\
\cline { 2 - 8 } & NIS & 262.74 & 265 & 304.22 & 165.28 & 310 & 348.38 \\
\hline
\end{tabular}

The best advantage to use the exponential membership function is to provide the different choices to DM so that he/she can get a profitable solution. These choices can be given by taking the number of cases which contains the combination of shape parameter and aspiration level. We expressed the outcomes for every choice of aspiration level and shape parameter appeared in Table 2. Here, we involved six cases only, more cases are also possible according to the DM's desire. 
International Journal of Mathematical, Engineering and Management Sciences

Vol. 5, No. 6, 1452-1467, 2020

https://doi.org/10.33889/IJMEMS.2020.5.6.108

Table 2. Different cases by DM

\begin{tabular}{|c|c|c|}
\hline Case & Shape parameters & Aspiration levels \\
\hline 1 & $(-1,-2)$ & $0.7,0.75$ \\
2 & $(-2,-1)$ & $0.85,0.7$ \\
3 & $(-1,-5)$ & $0.8,07$ \\
4 & $(-5,-1)$ & $0.75,0.8$ \\
5 & $(-2,-5)$ & $0.7,0.7$ \\
6 & $(-5,-2)$ & $0.8,0.8$ \\
\hline
\end{tabular}

Table 3. Summary results for $\alpha=0.1$

\begin{tabular}{|c|c|c|c|c|}
\hline Case & $\prod_{\mathbf{k}=\mathbf{1}}^{2} \prod_{\mathbf{i}=\mathbf{1}}^{3} \mu_{\mathbf{k i}}(\mathbf{x})$ & $\begin{array}{c}\text { Degree of } \\
\text { Satisfaction }\end{array}$ & Membership function value & Objective Value \\
\hline 1 & 0.5604 & 0.8192 & $\begin{array}{c}(0.843,0.8192,0.8578) \\
(0.9822,0.9815,0.9814)\end{array}$ & $\begin{array}{c}(144.5,176,197.56) \\
(158.89,175,196.23)\end{array}$ \\
\hline 2 & 0.6632 & 0.8877 & $\begin{array}{c}(0.9042,0.8877,0.9142) \\
(0.9678,0.9665,0.9663)\end{array}$ & $\begin{array}{c}(144.5,176,197.56) \\
(158.89,175,196.23)\end{array}$ \\
\hline 3 & 0.8342 & 0.9343 & $\begin{array}{c}(0.9343,0.9346,0.9993) \\
(0.9819,0.9853,0.9881)\end{array}$ & $\begin{array}{c}(129.9,156,173.16) \\
(187.04,200,217.38)\end{array}$ \\
\hline 4 & 0.8607 & 0.9663 & $\begin{array}{c}(0.9844,0.9806,0.9865) \\
(0.9678,0.9665,0.9663)\end{array}$ & $\begin{array}{c}(144.5,176,197.56) \\
(158.89,175,196.23)\end{array}$ \\
\hline 5 & 0.8836 & 0.9627 & $\begin{array}{c}(0.9627,0.9628,0.9996) \\
(0.9807,0.9853,0.9869)\end{array}$ & $\begin{array}{c}(129.9,156,173.16) \\
(188.336,200,219.12)\end{array}$ \\
\hline 6 & 0.9009 & 0.9806 & $\begin{array}{c}(0.9844,0.9806,0.9865) \\
(0.9822,0.9815,0.9814)\end{array}$ & $\begin{array}{c}(144.5,176,197.56) \\
(158.89,175,196.23)\end{array}$ \\
\hline
\end{tabular}

Table 4. Summary results for $\alpha=0.5$

\begin{tabular}{|c|c|c|c|c|}
\hline Case & $\prod_{\mathbf{k}=1}^{2} \prod_{i=1}^{3} \mu_{\mathbf{k i}}(\mathbf{x})$ & $\begin{array}{c}\text { Degree of } \\
\text { Satisfaction }\end{array}$ & Membership function value & Objective Value \\
\hline 1 & 0.561 & 0.8771 & $\begin{array}{c}(0.9001,0.9019,0.9784) \\
(0.8771,0.8902,0.9046)\end{array}$ & $\begin{array}{c}(147.3,162,183.6) \\
(198.835,205,230.25)\end{array}$ \\
\hline 2 & 0.643 & 0.8877 & $\begin{array}{c}(0.8952,0.8877,0.9108) \\
(0.9633,0.9665,0.954)\end{array}$ & $\begin{array}{c}(158.5,176,205.4) \\
(166.94,175,206.85)\end{array}$ \\
\hline 3 & 0.835 & 0.9337 & $\begin{array}{c}(0.9633,0.9665,0.954) \\
(0.9829,0.9853,0.9875)\end{array}$ & $\begin{array}{c}(141.5,156,179.4) \\
(193.52,200,226.07)\end{array}$ \\
\hline 4 & 0.843 & 0.9541 & $\begin{array}{c}(0.9824,0.9806,0.9858) \\
(0.9824,0.9806,0.9858)\end{array}$ & $\begin{array}{c}(158.5,176,205.4) \\
(166.94,175,206.85)\end{array}$ \\
\hline 5 & 0.886 & 0.9623 & $\begin{array}{c}(0.9623,0.9628,1) \\
(0.9829,0.9853,0.9875)\end{array}$ & $\begin{array}{c}(141.5,156,179.4) \\
(193.52,200,226.07)\end{array}$ \\
\hline 6 & 0.89 & 0.9743 & $\begin{array}{c}(0.9824,0.9806,0.9858) \\
(0.9796,0.9815,0.9743)\end{array}$ & $\begin{array}{c}(158.5,176,205.4) \\
(166.94,175,206.85)\end{array}$ \\
\hline
\end{tabular}

Here, we use triangular possibilistic distribution and take three $\alpha$-level, $\alpha=0.1, \alpha=0.5$ and $\alpha=0.9$. Corresponding to each $\alpha$ and each above six cases contain the combination of shape parameter and aspiration level, the Table 3, Table 4 and Table 5 represents the values of the objective function, corresponding membership value, degree of satisfaction and the final product operator value. These Tables shows that the objective value is different for different cases, it gives the different choices to DM so that he/she can take the batter decision. It is also shown that the change in shape parameter, influence the degree of satisfaction level for each objective function and gives more choices to DM. 
International Journal of Mathematical, Engineering and Management Sciences

Vol. 5, No. 6, 1452-1467, 2020

https://doi.org/10.33889/IJMEMS.2020.5.6.108

Table 5. Summary results for $\alpha=0.9$

\begin{tabular}{|c|c|c|c|c|}
\hline Case & $\prod_{\mathbf{k}=1}^{2} \prod_{\mathbf{i}=1}^{3} \mu_{\mathbf{k i}}(\mathbf{x})$ & $\begin{array}{c}\text { Degree of } \\
\text { Satisfaction }\end{array}$ & Membership function value & Objective Value \\
\hline 1 & 0.663 & 0.9062 & $\begin{array}{c}(0.9355,0.9346,1) \\
(0.9062,0.9082,0.9212)\end{array}$ & $\begin{array}{c}(153.1,156,185.64) \\
(198.704,200,233.02)\end{array}$ \\
\hline 2 & 0.637 & 0.8877 & $\begin{array}{c}(0.8915,0.8877,0.9072) \\
(0.9659,0.9665,0.9504)\end{array}$ & $\begin{array}{c}(172.5,176,213.24) \\
(173.389,175,215.34)\end{array}$ \\
\hline 3 & 0.758 & 0.9019 & $\begin{array}{c}(0.9033,0.902,0.9815) \\
(0.9806,0.981,0.9852)\end{array}$ & $\begin{array}{c}(159.06,162,189.36) \\
(203.767,205,236.98)\end{array}$ \\
\hline 4 & 0.841 & 0.9504 & $\begin{array}{c}(0.9815,0.9806,0.9851) \\
(0.9659,0.9665,0.9504)\end{array}$ & $\begin{array}{c}(172.5,176,213.24) \\
(173.389,175,215.34)\end{array}$ \\
\hline 5 & 0.889 & 0.9628 & $\begin{array}{c}(0.9634,0.9628,1) \\
(0.9848,0.9853,0.988)\end{array}$ & $\begin{array}{c}(153.1,156,185.64) \\
(198.704,200,233.02)\end{array}$ \\
\hline 6 & 0.888 & 0.9722 & $\begin{array}{c}(0.9815,0.9806,0.9851) \\
(0.9811,0.9815,0.9722)\end{array}$ & $\begin{array}{c}(172.5,176,213.24) \\
(173.389,175,215.34)\end{array}$ \\
\hline
\end{tabular}

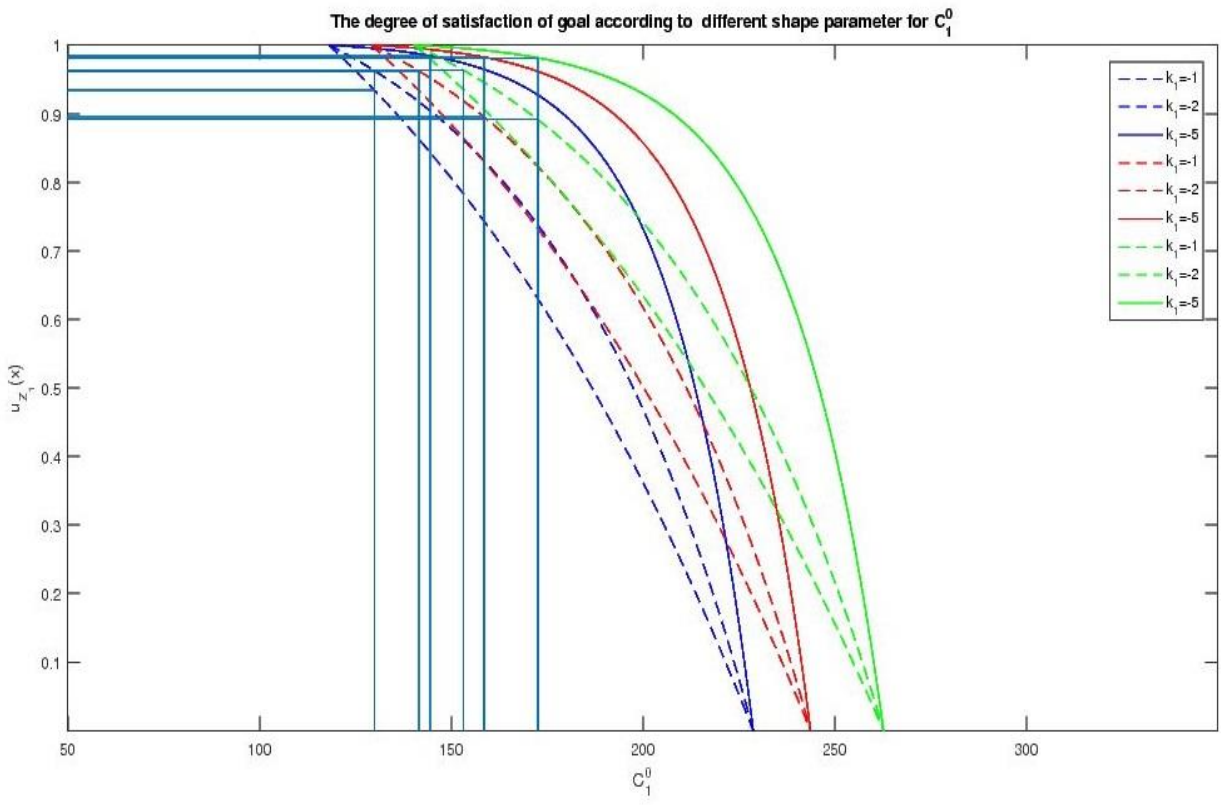

Figure 2. The degree of satisfaction according to different shape parameter for $\alpha=0.1$
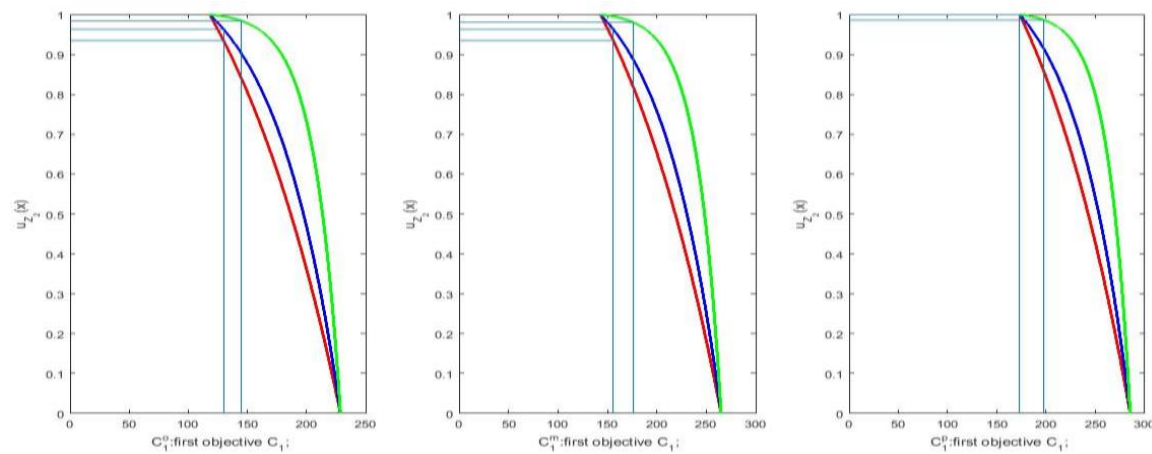

Figure 3. The degree of satisfaction of the first objective for $\alpha=0.1$ 
International Journal of Mathematical, Engineering and Management Sciences

Vol. 5, No. 6, 1452-1467, 2020

https://doi.org/10.33889/IJMEMS.2020.5.6.108
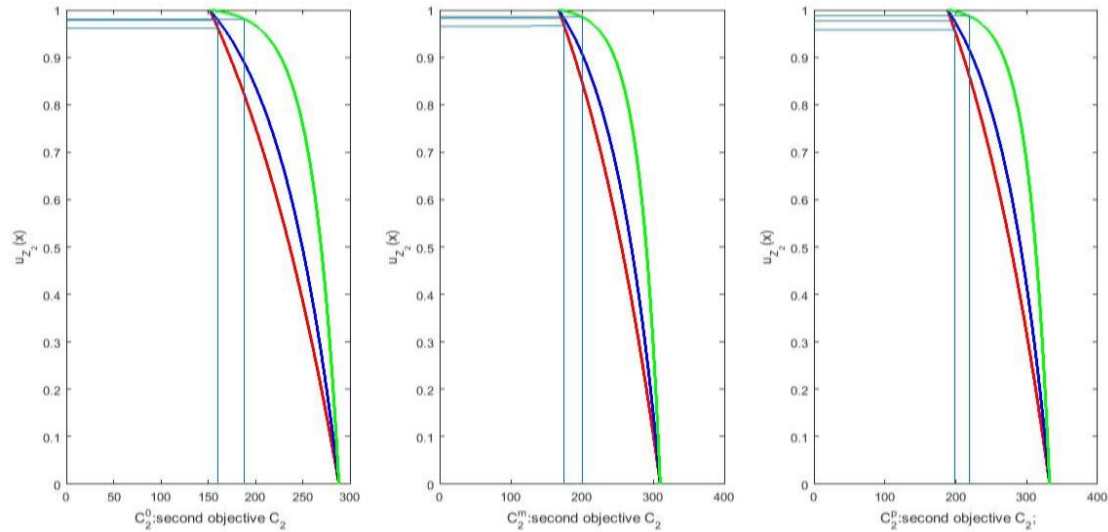

Figure 4. The degree of satisfaction of the second objective for $\alpha=0.1$

Corresponding to case-2, Figure 2 shows the degree of satisfaction for the first objective with optimistic case corresponding to $\alpha=0.1$. Figure 3 and Figure 4 respectively shows the distinction of the first objective value and second objective value corresponding to $(-1,-2,-5)$ shape parameter for optimistic case.

Figure 5 shows the obtained solution of the given FMOTP for different possibilistic distribution corresponding to the given cases in Table 2 . These Figures clearly show that with increasing the value of $\alpha$-cut manipulate of uncertainty decreases. Additionally, the use of exponential membership function, shape parameter and aspiration level can be confirmed. Moreover, if the solution is not favourable to DM, he may add one or more combination and get the solution accordingly.
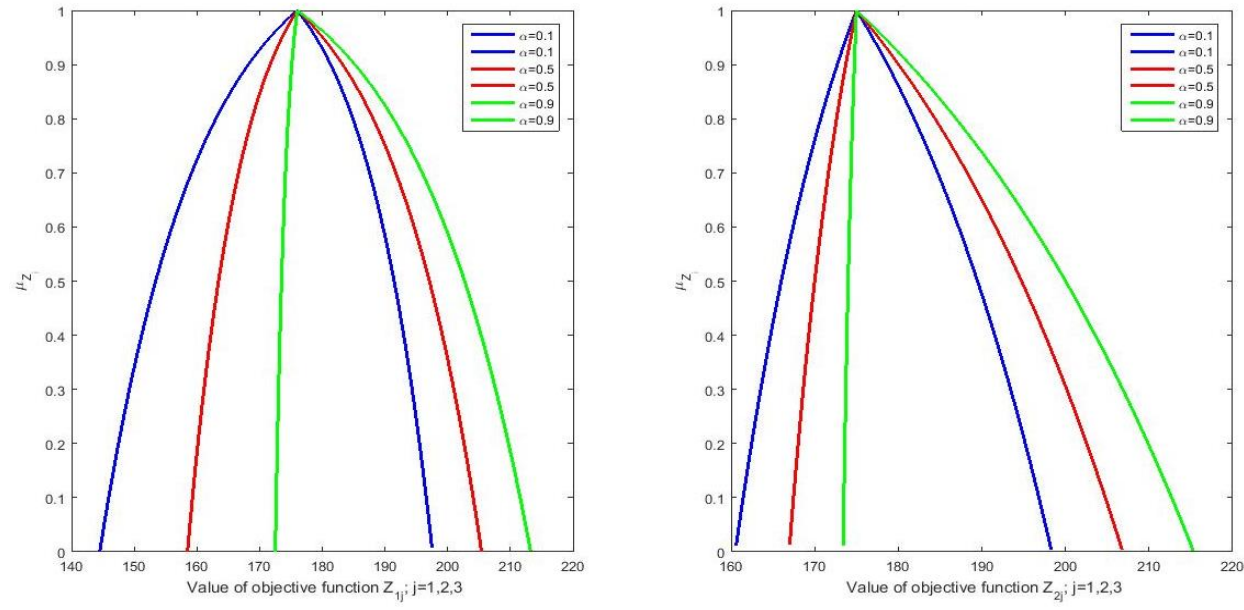

Figure 5. Possibilities distribution of first and second objectives for case-2 
International Journal of Mathematical, Engineering and Management Sciences

Vol. 5, No. 6, 1452-1467, 2020

https://doi.org/10.33889/IJMEMS.2020.5.6.108

Corresponding to different $\alpha$-cut, the solution of FMOTP for the given combination of shape parameter and aspiration level is represented in Figure 6. From this Figure, we conclude that in fuzzy judgment the impact of uncertainty decreases, with the value of $\alpha$ increasing.

\subsection{The Convergence Rate of GA}

The solution of the above FMOTP for $\alpha=0.1$ levels was obtained for case- 5 as shown in Figure 6 . The degree of satisfaction obtained after 20 iterations and 10 or more than 10 populations (or after 40 populations and 5 or more than 5 iterations) for $\alpha=0.1$. Similarly, the degree of satisfaction obtained after 15 iterations and 10 or more than 10 populations (or after 30 populations and 5 or more than 5 iterations) for $\alpha=0.5$ and the same way the degree of satisfaction for $\alpha=0.9$ can be observed.
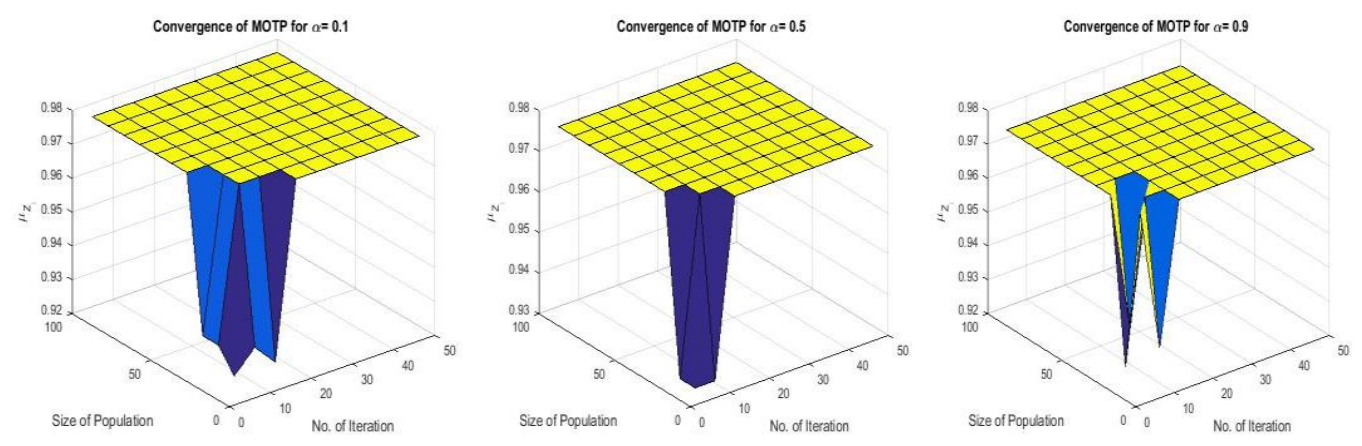

Figure 6. Convergence to the optimum solution for FMOTP at $\alpha=0.1,0.5,0.9$

\subsection{Simulation}

The computational results and corresponding efficient solution are represented in Table 3 for $\alpha=0.1$, $\alpha=0.5$ and $\alpha=0.9$. From the Table, it is clear, according to the different cases the values of objective functions were also different. These different values for different combination give the choice of the desired goal of an efficient solution. Here, we consider only some cases of shape parameter and aspiration level, however, if the DM wants he/she can add more cases for their desired goal i.e. if the DM is not happy with the obtained solution, more solution can be obtained by adding the cases in above Table 2 to improve the objective function according to the DM's satisfaction. Thus, the above Tables make known that the GA based hybrid approach can handle the FMOTP with the desired degree of satisfaction that gives more choice to DM. According to Mahapatra et al. (2010) membership function is used for describing the overall performance of indistinct data, usage of the fuzzy numbers of DM, options towards uncertainty, etc. With different membership function, the degree of satisfaction can be obtained and all provided an efficient solution. Mahapatra et al. (2010) suggest that linear membership function commonly used as its miles clean to implement as it defined through two fixing factors that are maximum value and minimum value. Exponential membership function reflects the reality better than linear membership as the marginal rate of increase of membership values as a function of the model parameter is not constant in such circumstances, the exponential membership function gives healthier demonstration then others and provides the flexibility to express grade of exactness in parameter values.

The graphical representation of the obtained solutions for different $\alpha$ level was represented in the above Figures. The triangular number is the case of the fuzzy number. The possibilistic distribution 
International Journal of Mathematical, Engineering and Management Sciences

Vol. 5, No. 6, 1452-1467, 2020

https://doi.org/10.33889/IJMEMS.2020.5.6.108

of first and second objective was represented in the Figure 5. The distribution suggests higher the value of $\alpha$, more reliable solution to DM was obtained.

\section{Comparison}

Table 6. Comparison with other approach

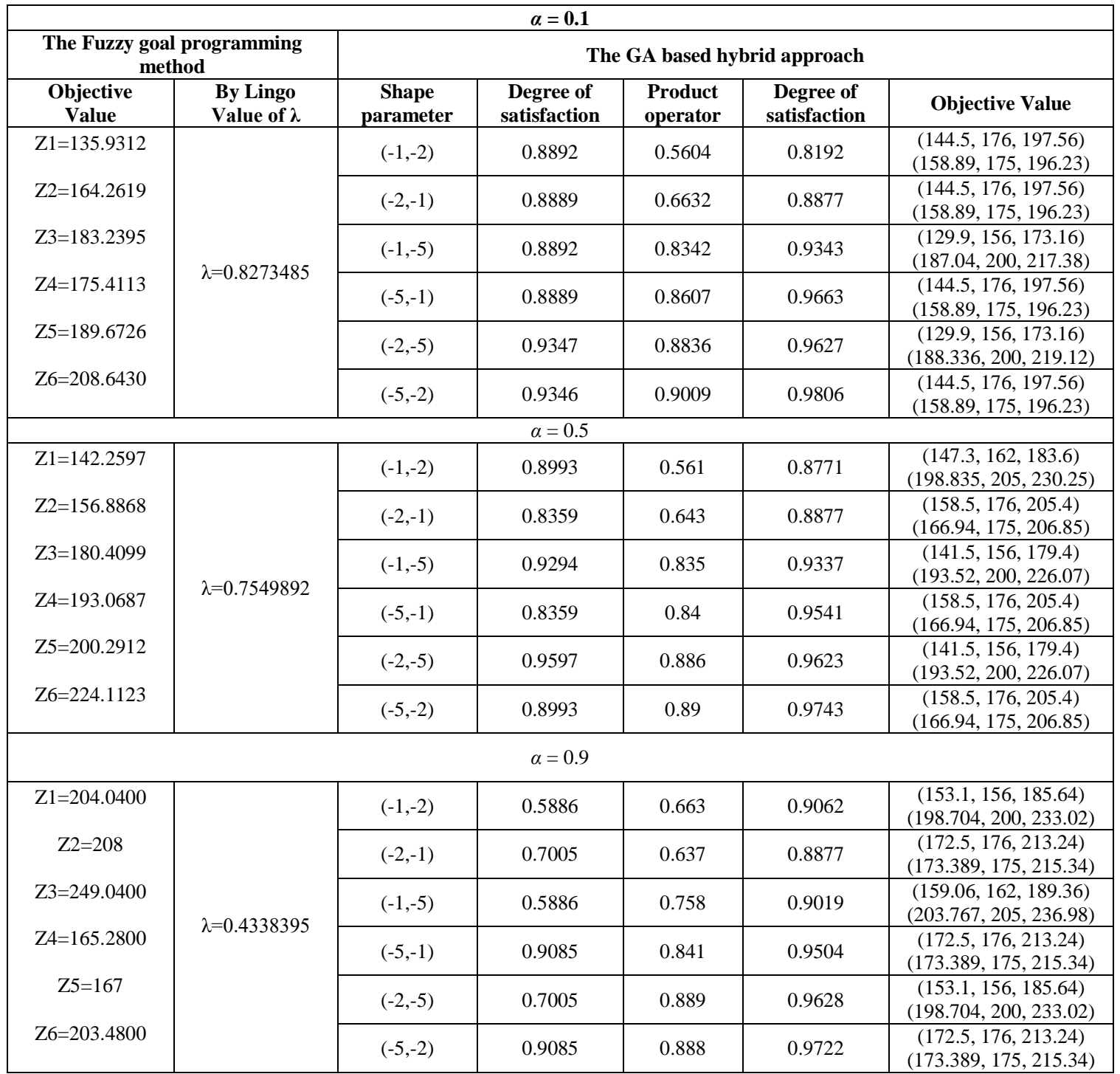

Above Table 6 shows the comparison between fuzzy goal programming method and GA based hybrid method for the model of fuzzy multi-objective transportation problem at $\alpha=0.1, \alpha=0.5$ and $\alpha=0.9$. The Table shows that with fuzzy goal programming method solution cannot be obtain according to the preference of DM also there is only single solution of the given problem and it's the only choice of DM, while in other side with the help of GA based hybrid approach, we can give more choices to DM. 
International Journal of Mathematical, Engineering and Management Sciences

Vol. 5, No. 6, 1452-1467, 2020

https://doi.org/10.33889/IJMEMS.2020.5.6.108

\section{Conclusion}

The proposed approach obtains the best effective solution of the given FMOTP. Moreover, with the help of exponential membership function higher degree of satisfaction was obtained. Tabular form of the solution is given in accordance to different values of shape parameter and different estimates of aspiration levels. It is also shown that the change in shape parameter, influence the degree of satisfaction level for each objective function and all obtained solutions are reliable with the preference of DM.

\section{Conflict of Interest}

The authors declare that there is no conflict of interest for this publication.

\section{Acknowledgement}

I would like to express my special thanks of gratitude to my teacher, my institute and Journal reviewers and editors.

\section{References}

Abd El-Wahed, W.F. (2001). A multi-objective transportation problem under fuzziness. Fuzzy Sets and Systems, 117(1), 27-33.

Abd El-Wahed, W.F., \& Lee, S.M. (2006). Interactive fuzzy goal programming for multi-objective transportation problems. Omega, 34(2), 158-166.

Bit, A.K., Biswal, M.P., \& Alam, S.S. (1993). Fuzzy programming approach to multi-objective solid transportation problem. Fuzzy Sets and Systems, 57(2), 183-194.

Chanas, S., Kołodziejczyk, W., \& Machaj, A. (1984). A fuzzy approach to the transportation problem. Fuzzy Sets and Systems, 13(3), 211-221.

Charnes, A., \& Cooper, W.W. (1954). The stepping stone method of explaining linear programming calculations in transportation problems. Management Science, 1(1), 49-69.

Das, S.K., Goswami, A., \& Alam, S.S. (1999). Multi-objective transportation problem with interval cost, source and destination parameters. European Journal of Operational Research, 117(1), 100-112.

Dhodiya, J.M., \& Tailor, A.R. (2016). Genetic algorithm based hybrid approach to solve fuzzy multiobjective assignment problem using exponential membership function. Springer Plus, 5(1), 2028.

Garfinkel, R.S., Neebe, A.W., \& Rao, M.R. (1974). An algorithm for the m-median plant location problem. Transportation Science, 8(3), 217-236.

Gen, M., Li, Y., \& Ida, K. (1999). Solving multi-objective transportation problem by spanning tree-based genetic algorithm. IEICE TRANSACTIONS on Fundamentals of Electronics, Communications and Computer Sciences, 82(12), 2802-2810.

Gupta, P., \& Mehlawat, M.K. (2013). A new possibilistic programming approach for solving fuzzy multiobjective assignment problem. IEEE Transactions on Fuzzy Systems, 22(1), 16-34.

Hitchcock, F.L. (1941). The distribution of a product from several sources to numerous localities. Journal of Mathematics and Physics, 20(1-4), 224-230.

Jiménez, F., \& Verdegay, J.L. (1998). Uncertain solid transportation problems. Fuzzy Sets and Systems, 100(1-3), 45-57. 
International Journal of Mathematical, Engineering and Management Sciences

Vol. 5, No. 6, 1452-1467, 2020

https://doi.org/10.33889/IJMEMS.2020.5.6.108

Kantorovich, L.V. (1960). Mathematical methods of organizing and planning production. Management Science, 6(4), 366-422.

Lee, S.M., \& Moore, L.J. (1973). Optimizing transportation problems with multiple objectives. AIIE Transactions, 5(4), 333-338.

Li, L., \& Lai, K.K. (2000). A fuzzy approach to the multi-objective transportation problem. Computers \& Operations Research, 27(1), 43-57.

Mahapatra, D.R., Roy, S.K., \& Biswal, M.P. (2010). Stochastic based on multi-objective transportation problems involving normal randomness. Advanced Modelling and Optimization, 12(2), 205-223.

Maity, G., \& Roy, S.K. (2014). Solving multi-choice multi-objective transportation problem: a utility function approach. Journal of Uncertainty Analysis and Applications, 2(1), 11.

Osuji, G.A., Okoli Cecilia, N., \& Opara, J. (2014). Solution of multi-objective transportation problem via fuzzy programming algorithm. Science Journal of Applied Mathematics and Statistics, 2(4), 71-77.

Rani, D., \& Gulati, T.R. (2016). Uncertain multi-objective multi-product solid transportation problems. Sādhanā, 41(5), 531-539.

Tabassum, M., \& Mathew, K. (2014). A genetic algorithm analysis towards optimization solutions. International Journal of Digital Information and Wireless Communications, 4(1), 124-142.

Verma, R., Biswal, M.P., \& Biswas, A. (1997). Fuzzy programming technique to solve multi-objective transportation problems with some non-linear membership functions. Fuzzy Sets and Systems, 91(1), 3743.

Zadeh, L.A. (1975). The concept of a linguistic variable and its application to approximate reasoning-III. Information Sciences, 9(1), 43-80.

Zimmermann, H.J. (1978). Fuzzy programming and linear programming with several objective functions. Fuzzy Sets and Systems, 1(1), 45-55. 